\title{
A computation of the maximal order type of the term ordering on finite multisets.
}

\author{
Andreas Weiermann ${ }^{\star}$ \\ University of Ghent \\ Vakgroep Zuivere Wiskunde en Computeralgebra \\ Krijgslaan 281 Gebouw S22 \\ 9000 Ghent \\ Belgium \\ Andreas. Weiermann@ugent . be
}

\begin{abstract}
We give a sharpening of a recent result of Aschenbrenner and Pong about the maximal order type of the term ordering on the finite multisets over a wpo. Moreover we discuss an approach to compute maximal order types of well-partial orders which are related to tree embeddings.
\end{abstract}

Keywords: well-quasi orderings, well-partial orderings, maximal order types, ordinals, term orderings, finite multisets

\section{Introduction}

A well-partial order (wpo) is a partial order $\left\langle X, \leq_{X}\right\rangle$ such that for all infinite sequences $\left\{x_{i}\right\}_{i=0}^{\infty}$ of elements in $X$ there exist natural numbers $i, j$ such that $i<j$ and $x_{i} \leq_{X} x_{j}$. There are lots of examples for wpo's known, for example well-orders or wpo's resulting from Higman's Lemma and Kruskal's theorem. Well-partial orderings (and similarly well-quasi orderings) play an important role in logic, mathematics and computer science, since they form a convenient tool for proving termination of algorithms. For example the well-foundedness of syntactic termination orderings like the recursive path ordering follows easily by an appeal to Kruskal's theorem [6]. Famous applications of wpo's in logic are provided, for example, by Ehrenfeucht's well-foundedness proof for Skolem's ordering on extended polynomials [7] or the termination proof for decision procedures related to relevance logic [18].

In mathematics wpo's are, for example, used to show termination of the algorithm which computes Gröbner bases in polynomial rings [2]. An obvious question related to such termination proofs for algorithms is the question about resulting running times of the algorithms.

\footnotetext{
* The author acknowledges gratefully funding by the John Templeton Foundation, the Fonds voor Wetenschappelijk Onderzoek Vlaanderen (FWO) and (via a joint project with Michel Rathjen) funding by the Royal Society (UK).
} 
From the folklore of subrecursive hierarchy theory it is known that as a rule of thumb such running times are bounded by $\alpha$-descent recursive functions where $\alpha$ is the maximal order type of the underlying wpo [3]. This is particularly interesting if the maximal order type in question is small.

Therefore let us explain maximal order types and parts of their history in more detail. An early but very fundamental result concerning maximal order types is the following result of de Jongh and Parikh [4]:

For every wpo $\left\langle X, \leq_{X}\right\rangle$ there exists a linear extension $\leq^{+}$of $\leq_{X}$ on the same set $X$ such that the order type is maximal possible under all such well-ordered extensions.

In this situation we put $o\left(X, \leq_{X}\right):=\operatorname{otype}\left(X, \leq^{+}\right)$and the ordinal $o\left(X, \leq_{X}\right)$ is then (for obvious reasons) called the maximal order type of $\langle X, \leq\rangle$. (In the sequel we write $\leq$ for $\leq_{X}$ when there is no danger of confusion.) The result by de Jongh and Parikh can be used to actually compute maximal order types using the following formula:

$$
o(X, \leq)=\sup \left\{o\left(L_{X}(x), \leq\right)+1: x \in X\right\}
$$

where $L_{X}(x):=\{y \in X: \neg x \leq y\}$.

In logic, and proof theory in particular, maximal order types are typically the invariants which determine the proof theoretic strength of an assertion that a given poset is a wpo. As a rule of thumb (see, for example, [13] for a paradigm) one (quite often) observes the following principle: If it is possible to calculate the maximal order type of a wpo $X$ in terms of an ordinal notation system then the assertion that the poset $X$ is a wpo is over $\mathrm{ACA}_{0}$ equivalent to the assertion that the linearly ordered ordinal terms below the term representing $o(X)$ form a well-order.

There are several results known about maximal order types, and important sources for maximal order types are still [4] and the (unpublished) Habilitationsschrift of Diana Schmidt [15].

Some rudiments of wpo-theory are as follows. Suppose we have given two posets $X_{0}$ and $X_{1}$. Then we can define induced partial orders on the disjoint union $X_{0} \oplus X_{1}$ and the cartesian product $X_{0} \otimes X_{1}$ in the natural way. Moreover the set $X^{*}$ of finite sequences of elements over $X$ can be partially ordered using the natural pointwise ordering induced on subsequences (Higman ordering). With $\oplus$ and $\otimes$ we denote the (commutative) natural sum and the (commutative) natural product of ordinals.

Theorem 1 (De Jongh and Parikh [4], Schmidt [15]) 1. If $X_{0}$ and $X_{1}$ are wpo's then $X_{0} \oplus X_{1}$ and $X_{0} \otimes X_{1}$ are wpo's, o $\left(X_{0} \oplus X_{1}\right)=o\left(X_{0}\right) \oplus o\left(X_{1}\right)$ and $o\left(X_{0} \otimes X_{1}\right)=o\left(X_{0}\right) \otimes o\left(X_{1}\right)$.

2. If $X$ is a wpo then $X^{*}$ is a wpo and the following cases occur:

$$
o\left(X^{*}\right)= \begin{cases}\omega^{\omega^{o(X)-1}} & \text { if } X \text { is finite, } \\ \omega^{\omega^{o(X)+1}} & \text { if } o(X)=\epsilon+n \text { where } \epsilon \text { is an epsilon number } \\ & \text { and } n \text { is finite, } \\ \omega^{\omega^{o(X)}} & \text { otherwise. }\end{cases}
$$


Quite recently wpo's related to monomial ideals have been investigated in detail by M. Aschenbrenner and W. Y. Pong [1]. Among other things they provided bounds on maximal order type of the term ordering on finite multisets. The aim of this paper is to provide a precise formula for such a maximal order type.

We intend to use this specific result in future investigations on maximal order types emerging from well-partial orderings related to tree-embeddability relations which are based on a Friedman style gap condition [17].

To this end we recently developed (in a joint research project with Michael Rathjen) a very satisfying and general formula which predicts in all natural cases (at which we had a look at) good upper bounds for the maximal order type of a tree-based wpo under consideration [21].

To explain this formula informally let us recall briefly the definition of the collapsing function needed for a proof-theoretic analysis of $I D_{1}$ (see, for example, [14] for an exposition). Let $\Omega$ denote the first uncountable ordinal and $\varepsilon_{\Omega+1}$ the first epsilon number above $\Omega$. Then recall that any ordinal $\alpha<\varepsilon_{\Omega+1}$ can be described uniquely in terms of its Cantor normal form

$$
\alpha=\Omega^{\alpha_{1}} \beta+\cdots+\Omega^{\alpha_{n}} \cdot \beta_{n}
$$

where $\alpha_{1}>\ldots>\alpha_{n}$ and $0<\beta_{1}, \ldots, \beta_{n}<\Omega$. In this situation we define the countable subterms $K \alpha$ of $\alpha$ recursively via

$$
K \alpha:=K \alpha_{1} \cup \ldots \cup K \alpha_{n} \cup\left\{\beta_{1}, \ldots, \beta_{n}\right\}
$$

where $K 0:=0$. Let $A P=\left\{\omega^{\delta}: \delta \in O N\right\}$. We can then put

$$
\vartheta \alpha:=\min \{\beta \in A P: \beta \geq \max K \alpha \wedge \forall \gamma<\alpha(K \gamma<\beta \rightarrow \vartheta \gamma<\beta\} .
$$

One easily verifies $\vartheta \alpha<\Omega$ by induction on $\alpha$ using a cardinality argument. It is moreover easy to verify that then $\varepsilon_{0}=\vartheta \Omega$ and $\Gamma_{0}=\vartheta \Omega^{2}$ (see, for example, [13].)

To explain the expected formula concerning wpo's let us consider a given explicit operator $W$ which maps a (countable) wpo $X$ to a (countable) wpo $W(X)$ so that the elements of $W(X)$ can be described as generalized terms in which the variables are replaced by constants for the elements of $X$. We assume that the ordering between elements of $W(X)$ is induced effectively by the ordering from $X$. (This resembles Feferman's notion of effective relative categoricity [8], Girard's notion of denotation system [10] or Joyal's notion of analytic functor. The latter notion seems to contain Feferman's and Girard's notions, as indicated e.g. in [11].) In concrete situations $W$ may for example stand for an iterated application of basic constructions like disjoint union and cartesian product, the set of finite sequences construction, the multiset construction, or a tree constructor and the like. We assume that for $W$ we have an explicit knowledge of $o(W(X))$ such that $o(W(X))=o(W(o(X)))$ and such that this equality can be proved using an effective reification (An example for this technique is, for example, given in $[13]$ or $[16])$.

Using $W$ we then build the set of $W$-constructor trees $T(W(\operatorname{Rec}))$ as follows: 
1. $\cdot \in T(W(R e c))$.

2. If $\left(s_{i}\right)$ is a sequence of elements in $T(W(\operatorname{Rec}))$ and $w\left(\left(x_{i}\right)\right)$ is a term from $W(X)$ then $\cdot\left(w\left(\left(s_{i}\right)\right)\right) \in T(W(\operatorname{Rec}))$.

The embeddability relation $\unlhd$ on $T(W(R e c))$ is defined recursively as follows:

1. $\cdot \triangleleft t$.

2. If $s \unlhd t_{i}$ then $s \unlhd \cdot\left(w\left(\left(t_{i}\right)\right)\right)$

3. If $\left.w\left(\left(s_{i}\right)\right) \leq w^{\prime}\left(\left(t_{j}\right)\right) \bmod W(T(W(\operatorname{Rec})))\right)$ is induced recursively by $\unlhd$ then

$$
\cdot\left(w\left(\left(s_{i}\right)\right)\right) \unlhd \cdot\left(w^{\prime}\left(\left(t_{j}\right)\right)\right) .
$$

The general principle is now that

$$
T(W(R e c)) \text { is a wpo }
$$

and

$$
o(\langle T(W(\operatorname{Rec})), \unlhd\rangle) \leq \vartheta o(W(\Omega))
$$

for $o(W(\Omega)) \in \operatorname{dom}(\vartheta)$ with $o(W(\Omega)) \geq \Omega^{3}$. [Moreover the reverse inequality follows in many cases by direct inspection.]

The formula (2) is true for several natural examples which appear as suborderings of Friedman's $F K T^{n}$ [17] (provided that when necessary the domain of $\vartheta$ is suitably extended, but discussing such matters is beyond the scope of the present article). We believe that (2) will be the key property in finally analyzing Friedman's $F K T^{n}$ and we have already obtained far reaching applications.

In general (2) can be proved along the following general outline. (This outline applies to all cases which we considered so far.)

Proof ("Proof outline" for (2)). The inequality is proved by induction on $o(W(\Omega)$ ). Let $t=w\left(\left(t_{j}\right)\right) \in T(W(\operatorname{Rec}))$. We claim $o\left(L_{T(W(R e c))}(t)\right)<\vartheta o(W(\Omega))$ and may assume by induction hypothesis that

$$
o\left(L_{T(W(R e c))}\left(t_{j}\right)\right)<\vartheta o(W(\Omega)) .
$$

If now $s \in L_{T(W(R e c))}(t)$ then there will be natural quasi-embedding putting $s$ into a well-partial order $W^{\prime}\left(R e c,\left(t_{i}\right)\right)$ such that

$$
o\left(W^{\prime}\left(\Omega,\left(t_{i}\right)\right)\right)<o(W(\Omega))
$$

and such that

$$
K\left(o\left(W^{\prime}\left(\Omega,\left(t_{i}\right)\right)\right)\right) \leq \max \left(K\left(o(W(\Omega)) \cup\left\{o\left(L_{T(W(R e c))}\left(t_{j}\right)\right): j\right\}\right)\right.
$$

This step uses the assumption that the maximal order type resulting from $W$ can be computed by an effective reification a la [13] or [16]. Therefore the definition of $\vartheta$ yields

$$
\vartheta\left(o\left(W^{\prime}\left(\Omega,\left(t_{i}\right)\right)\right)\right)<\vartheta(o(W(\Omega))) .
$$

By induction hypothesis

$$
o\left(L_{T(W(R e c))}(t)\right) \leq o\left(T\left(W^{\prime}\left(\Omega,\left(t_{i}\right)\right)\right)\right) \leq \vartheta\left(o\left(W^{\prime}\left(\Omega,\left(t_{i}\right)\right)\right)\right)
$$

and we are done. 
This proof outline can be used to prove (rigorously) several of the main results of the Habilitationsschrift of Diana Schmidt [15] in a short and uniform way, but there already have been lots of more applications (which exceed the realm of the usual Kruskal theorem).

Examples 1 (Rathjen and Weiermann) 1. If $W(X)=X^{*}$ then $o(\langle T(W($ Rec $)), \unlhd\rangle)=\vartheta \Omega^{\omega}\left(\right.$ since $\left.o\left(\Omega^{*}\right)=\omega^{\omega^{\Omega+1}}=\Omega^{\omega}\right)$.

2. If $W(X)=\bigotimes_{i<n} X$ then $o(\langle T(W(R e c)), \unlhd\rangle)=\vartheta \Omega^{n} \quad$ (since $o\left(\bigotimes_{i<n} \Omega\right)=$ $\left.\Omega^{n}\right)$.

3. If $W(X)=\left(X^{*}\right)^{*}$ then $o(\langle T(W(\operatorname{Rec})), \unlhd\rangle)=\vartheta \Omega^{\Omega^{\Omega^{\omega}}} \quad\left(\right.$ since $o\left(\left(\Omega^{*}\right)^{*}\right)=$ $\left.\omega^{\omega^{\omega^{\omega^{\Omega+1}}}}=\Omega^{\Omega^{\Omega^{\omega}}}\right)$.

Further examples arise from the multiset construction. Let $M^{\prime}(X)$ be the set of finite multisets over $X$ ordered by (cf., e.g., [19])

$$
m \ll m^{\prime} \Longleftrightarrow\left(\forall x \in m \backslash m \cap m^{\prime}\right)\left(\exists y \in m^{\prime} \backslash m \cap m^{\prime}\right)[x<y] .
$$

Further let $B(X)$ be the set of binary (planar) trees labeled with elements from $X$ ordered under homeomorphic embeddability.

Examples 2 (Rathjen and Weiermann) 1. If $W(X)=M^{\prime}(X)$ then $o(\langle T(W(\operatorname{Rec})), \unlhd\rangle) \leq \vartheta \Omega\left(\right.$ since $\left.o\left(M^{\prime}(\Omega)\right)=\omega^{\Omega}=\Omega\right)$.

2. If $W(X)=M^{\prime}(X \otimes X)$ then $o(\langle T(W(R e c)), \unlhd\rangle)=\vartheta \Omega^{\Omega}$ (since $o\left(M^{\prime}(\Omega \otimes\right.$ $\left.\Omega))=\omega^{\Omega \otimes \Omega}=\Omega^{\Omega}\right)$.

3. If $W(X)=B(X)$ then $o(\langle T(W(R e c)), \unlhd\rangle)=\vartheta \varepsilon_{\Omega+1} \quad($ since $o(B(\Omega))=$ $\left.\varepsilon_{\Omega+1}\right)$.

Finally let $M(X)$ be the set of finite multisets over $X$ but now according to [1] ordered by

$$
m \leq^{\diamond} m^{\prime} \Longleftrightarrow\left(\exists f: m \hookrightarrow m^{\prime}\right)(\forall x \in m)[x \leq f(x) \bmod X] .
$$

The main result proved in this paper is then the following. Given $\alpha$ let

$$
\alpha^{\prime}:=\left\{\begin{array}{l}
\alpha+1 \text { if } \alpha \text { is an epsilon number } \\
\alpha \text { if } \alpha \text { is not an epsilon number. }
\end{array}\right.
$$

Theorem 2 If $o(X)=\omega^{\alpha_{1}}+\cdots+\omega^{\alpha_{n}} \geq \alpha_{1} \geq \ldots \geq \alpha_{n}$ then

$$
o(M(X))=\omega^{\omega^{\alpha_{1}{ }^{\prime}}+\cdots+\omega^{\alpha_{n}{ }^{\prime}} .} .
$$

This result and the formula (2) lead in many natural cases to the correct maximal order types for wpo's resulting from non planar trees since, e.g., $o(M(\Omega))=$ $\Omega^{\omega}$ and $o\left(T(M(\operatorname{Rec}))=\vartheta \Omega^{\omega}\right.$ [13]. Moreover since $o\left(M\left(\varepsilon_{0} \otimes \Omega\right)\right)=\omega^{\varepsilon_{0} \otimes \Omega}$ we find the result $o\left(\mathcal{T}\left(\begin{array}{l}1 \\ \varepsilon_{0}\end{array}\right)\right)=\vartheta\left(\Omega^{\varepsilon_{0}}\right)$ from [20]. Here the class $\mathcal{T}\left(\begin{array}{l}1 \\ \varepsilon_{0}\end{array}\right)$ from [20] corresponds to $T\left(M\left(\varepsilon_{0} \otimes \Omega\right)\right)$ in the current setting. 


\section{Proof of the Main Theorem}

Before proving our main result let us first recall a basic fact from wpo-theory which is useful for proving results on maximal order types.

Definition 1 Let $X, Y$ be two posets. A map e : $X \rightarrow Y$ is called a quasiembedding if for all $x, x^{\prime} \in X$ with $e(x) \leq e\left(x^{\prime}\right) \bmod Y$ we have $x \leq x^{\prime} \bmod$ $X$.

Lemma 1 If $X, Y$ are posets and $e: X \rightarrow Y$ is a quasi-embedding and $Y$ is a wpo, then $X$ is a wpo and $o(X) \leq o(Y)$.

Let us now come to our Main Theorem. Assume that $\langle X, \leq\rangle$ is a partial ordering and that $M(X)$ is the set of finite multisets over $X$. Recall the definition of the term ordering $\leq^{\diamond}$ [cf. (3)] and the definition of the operation $\alpha \mapsto \alpha^{\prime}$ [cf. (4)] from Sec. 1.

Main Theorem 1 Let $\langle X, \leq\rangle$ be a well-partial ordering with

$$
o(X)=\omega^{\alpha_{1}}+\cdots+\omega^{\alpha_{n}} \geq \alpha_{1} \geq \ldots \geq \alpha_{n}
$$

and let $M(X)$ be the set of finite multisets over $X$. Then

$$
o\left(M(X), \leq^{\diamond}\right)=\omega^{\omega^{\alpha_{1}{ }^{\prime}}+\cdots+\omega^{\alpha_{n}{ }^{\prime}}} .
$$

Proof. For $\alpha=\omega^{\alpha_{1}}+\cdots+\omega^{\alpha_{n}} \geq \alpha_{1} \geq \ldots \geq \alpha_{n}$ we write $\hat{\alpha}:=\omega^{\alpha_{1}{ }^{\prime}}+\cdots+\omega^{\alpha_{n}{ }^{\prime}}$. Then $\widehat{\alpha \oplus \beta}=\hat{\alpha} \oplus \hat{\beta}$. The proof of the inequality

$$
o\left(M(X), \leq^{\diamond}\right) \leq \hat{\alpha}
$$

is very similar to the proof provided by Aschenbrenner and Pong [1]. For convenience of the reader we recall the whole argument and we fill in the modifications when needed. The proof is by induction on $\alpha:=o(X)$. The case $\alpha=0$ is trivial and we may assume that $\alpha>0$. Now assume that $\alpha=\alpha_{1} \oplus \alpha_{2}$ where $\alpha_{1}, \alpha_{2}<\alpha$. Then $X$ is a disjoint union $X_{1} \cup X_{2}$ with $o\left(X_{1}\right) \leq \alpha_{1}$ and $o\left(X_{2}\right) \leq \alpha_{2}$. Then

$$
\begin{aligned}
o(M(X)) & \leq o\left(M\left(X_{1}\right) \otimes M\left(X_{2}\right)\right)=o\left(M\left(X_{1}\right) \otimes o\left(M\left(X_{2}\right)\right)\right. \\
& \leq \omega^{\hat{\alpha_{1}}} \otimes \omega^{\hat{\alpha_{2}}}=\omega^{\widehat{\alpha_{1} \oplus \alpha_{2}}}=\omega^{\hat{\alpha}}
\end{aligned}
$$

using the induction hypothesis. Now suppose that $\alpha=\omega^{\alpha_{1}}$. We may assume that $\alpha_{1}>0$.

It suffices to show that $o(L(w))<\omega^{\hat{\alpha}}$ for all $w \in M(X)$. We show this by induction on the length of $w$. If the length of $w$ is zero then we are done. Assume now that $w=\llbracket x_{0}, \ldots, x_{m-1} \rrbracket$ with $x_{0}, \ldots, x_{m-1} \in X$. Then there is a quasi-embedding $e: L(w) \rightarrow L_{X}\left(x_{0}\right) \oplus\left(X \otimes L\left(w^{\prime}\right)\right)$ where $w^{\prime}=\llbracket x_{1}, \ldots, x_{m-1} \rrbracket$. To see this let $v=\llbracket y_{0}, \ldots, y_{n-1} \rrbracket \in M(X)$ such that $\neg w \leq \diamond v$. Then either $\neg x_{0} \leq y_{i} \bmod X$ for all $i$; or $y_{i} \geq x_{0} \bmod X$ for some $i$, so after reordering the $y$ 's we may assume that $x_{0} \leq y_{0} \bmod X$ and $\neg w^{\prime} \leq^{\diamond} v^{\prime}$ for $v^{\prime}:=\llbracket y_{1}, \ldots, y_{n-1} \rrbracket$. 
In the first case we put $e(v):=v \in M\left(L\left(x_{0}\right)\right)$ and in the second case we put $e(v)=\left\langle y_{0}, v^{\prime}\right\rangle \in X \otimes L\left(w^{\prime}\right)$. It is easy to check that this is a quasi-embedding. Hence

$$
o(L(w)) \leq o\left(M\left(L\left(x_{0}\right)\right)\right) \oplus\left(\alpha \otimes o\left(L\left(w^{\prime}\right)\right)\right)
$$

by the previous Lemma. Put $\gamma=o\left(L\left(x_{0}\right)\right)$, then $\gamma<\alpha$ hence $\hat{\gamma}<\hat{\alpha}$ and $o\left(M\left(L\left(x_{0}\right)\right)\right) \leq \omega^{\hat{\gamma}}<\omega^{\hat{\alpha}}$ by induction hypothesis on $\alpha$. By induction hypothesis on $w$ we have $\delta:=o\left(L\left(w^{\prime}\right)\right)<\omega^{\hat{\alpha}}$. Hence it suffices to show that $\alpha \cdot \delta<\omega^{\hat{\alpha}}$. If $\alpha_{1}{ }^{\prime}=\alpha_{1}$ then $\alpha_{1}<\omega^{\omega^{\alpha_{1}}}$ and $\omega^{\omega^{\alpha_{1}}}$ is closed under natural multiplication. Hence $\delta<\omega^{\omega^{\alpha_{1}}}$ yields $\alpha \otimes \delta<\omega^{\hat{\alpha}}$. If $\alpha_{1}{ }^{\prime}>\alpha_{1}$ then $\alpha=\omega^{\omega^{\alpha_{1}}}<\omega^{\omega^{\alpha_{1}}}$ and $\omega^{\omega^{\alpha_{1}{ }^{\prime}}}$ is again closed under natural multiplication. Hence $\delta<\omega^{\hat{\alpha}}$ yields $\alpha \otimes \delta<\omega^{\hat{\alpha}}$. We are done in this case.

We now prove the other direction. Assume that $\alpha:=o(X)=\omega^{\alpha_{1}}+\cdots+\omega^{\alpha_{n}}$ where $\omega^{\alpha_{1}}+\cdots+\omega^{\alpha_{n}} \geq \alpha_{1} \geq \ldots \geq \alpha_{n}$. We claim that $o\left(M(X), \leq^{\diamond}\right) \geq \omega^{\hat{\alpha}}$. For this it suffices to show that $o(M(X), \preceq \diamond) \geq \omega^{\hat{\alpha}}$ where $\preceq$ is a linear extension of $\leq$ on $X$ having (maximal possible) order type $o(X)$. It is easy to see that $o\left(M(X), \leq^{\diamond}\right) \geq o\left(M(X), \preceq^{\diamond}\right)$ since any quasi-embedding $e: o(X) \rightarrow X$ gives rise to a corresponding quasi-embedding $\hat{e}: M(o(X)) \rightarrow M(X)$.

While proving

$$
o\left(M(X), \preceq^{\diamond}\right) \geq \omega^{\hat{\alpha}}
$$

we identify $X$ with $o(X)$, hence with $\alpha$.

Assume first that $\alpha_{1}$ is not an epsilon number. (This case will be considerably easier than the other.) We define a quasi-embedding $e: \omega^{o(X)} \rightarrow M(X)$ by

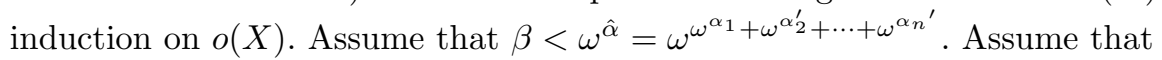

$$
\beta=\omega^{\omega^{\alpha_{1}}+\beta_{1}}+\cdots+\omega^{\omega^{\alpha_{1}}+\beta_{r}}+\omega^{\beta_{r+1}}+\cdots+\omega^{\beta_{r+s}}
$$

where $\beta$ is written in Cantor normal form and $\beta_{1}, \ldots, \beta_{r}<\omega^{\alpha_{2}{ }^{\prime}}+\cdots+\omega^{\alpha_{n}{ }^{\prime}}$

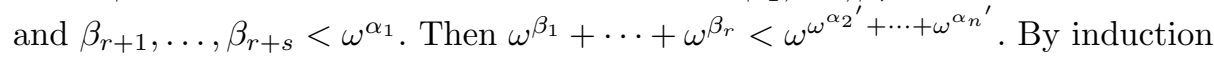
hypothesis we may assume that there is a quasi-embedding

$$
f: \omega^{\omega^{\alpha_{2}{ }^{\prime}}+\cdots+\omega^{\alpha_{n}{ }^{\prime}}} \rightarrow M\left(\omega^{\alpha_{2}}+\cdots+\omega^{\alpha_{n}}\right) .
$$

Assume that $f\left(\omega^{\beta_{1}}+\cdots+\omega^{\beta_{r}}\right)=\llbracket \delta_{1}, \ldots, \delta_{k} \rrbracket$.

Now put

$$
e(\beta)=\llbracket \omega^{\alpha_{1}}+\delta_{1}, \ldots, \omega^{\alpha_{1}}+\delta_{k} \rrbracket \cup \llbracket \beta_{r+1}, \ldots, \beta_{r+s} \rrbracket .
$$

Then $e(\beta) \in M(X)$. We claim that $e$ is indeed a quasi-embedding.

For, assume that $e(\beta) \leq^{\diamond} e(\gamma)$ with $\beta, \gamma<\omega^{o(\hat{X})}$. Write

$$
e(\beta)=\llbracket \omega^{\alpha_{1}}+\delta_{1}, \ldots, \omega^{\alpha_{1}}+\delta_{k} \rrbracket \cup \llbracket \beta_{r+1}, \ldots, \beta_{r+s} \rrbracket
$$

as before. Assume that

$$
\gamma=\omega^{\omega^{\alpha_{1}}+\gamma_{1}}+\cdots+\omega^{\omega^{\alpha_{1}}+\gamma_{t}}+\omega^{\gamma_{t+1}}+\cdots+\omega^{\gamma_{t+u}}
$$


where $\gamma$ is written in Cantor normal form and $\gamma_{1}, \ldots, \gamma_{t}<\omega^{\alpha_{2}{ }^{\prime}}+\cdots+\omega^{\alpha_{n}{ }^{\prime}}$ and $\gamma_{t+1}, \ldots, \gamma_{t+u}<\omega^{\alpha_{1}}$. Assume that $f\left(\omega^{\gamma_{1}}+\cdots+\omega^{\gamma_{t}}\right)=\llbracket \xi_{1}, \ldots, \xi_{l} \rrbracket$ and write

$$
e(\gamma)=\llbracket \omega^{\alpha_{1}}+\xi_{1}, \ldots, \omega^{\alpha_{1}}+\xi_{l} \rrbracket \cup \llbracket \gamma_{t+1}, \ldots, \gamma_{t+u} \rrbracket .
$$

From $e(\beta) \leq^{\diamond} e(\gamma)$ we conclude

$$
\llbracket \omega^{\alpha_{1}}+\delta_{1}, \ldots, \omega^{\alpha_{1}}+\delta_{k} \rrbracket \leq \llbracket \omega^{\alpha_{1}}+\xi_{1}, \ldots, \omega^{\alpha_{1}}+\xi_{l} \rrbracket \bmod \left(M\left(\omega^{\alpha_{2}}+\cdots+\omega^{\alpha_{n}}\right)\right)
$$

hence $f\left(\omega^{\beta_{1}}+\cdots+\omega^{\beta_{r}}\right) \leq f\left(\omega^{\gamma_{1}}+\cdots+\omega^{\gamma_{t}}\right)$ thus

$$
\omega^{\beta_{1}}+\cdots+\omega^{\beta_{r}} \leq \omega^{\gamma_{1}}+\cdots+\omega^{\gamma_{t}} .
$$

Multiplication by $\omega^{\omega^{\alpha_{1}}}$ on the left yields

$$
\omega^{\omega^{\alpha_{1}}+\beta_{1}}+\cdots+\omega^{\omega^{\alpha_{1}}+\beta_{r}} \leq \omega^{\omega^{\alpha_{1}}+\gamma_{1}}+\cdots+\omega^{\omega^{\alpha_{1}}+\gamma_{t}} .
$$

If the inequality would be strict, then $\beta<\gamma$ would follow immediately. So assume that

$$
\omega^{\omega^{\alpha_{1}}+\beta_{1}}+\cdots+\omega^{\omega^{\alpha_{1}}+\beta_{r}}=\omega^{\omega^{\alpha_{1}}+\gamma_{1}}+\cdots+\omega^{\omega^{\alpha_{1}}+\gamma_{t}} .
$$

Then

$$
\llbracket \beta_{r+1}, \ldots, \beta_{r+s} \rrbracket \leq \llbracket \gamma_{t+1}, \ldots, \gamma_{t+u} \rrbracket \bmod M\left(\omega^{\alpha_{1}}\right) .
$$

This yields $\omega^{\beta_{r+1}}+\cdots+\omega^{\beta_{r+s}} \leq \omega^{\gamma_{t+1}}+\cdots+\omega^{\gamma_{t+u}}$ hence $\beta \leq \gamma$.

Now we turn to the critical case that $\alpha_{1}$ is an epsilon number.

Let us assume that $o(X)=\epsilon+\tau$ where $\epsilon=\alpha_{1}$ and $\tau=\omega^{\alpha_{2}}+\cdots+\omega^{\alpha_{n}}$. Then $\hat{\epsilon}=\epsilon \cdot \omega$ and $\hat{\tau}=\omega^{\alpha_{2}^{\prime}}+\cdots+\omega^{\alpha_{n}{ }^{\prime}}$. We define $e: \omega^{\epsilon \cdot \omega+\hat{\tau}} \rightarrow M(X)$ as follows. Pick $\beta<\omega^{\epsilon \cdot \omega+\hat{\tau}}$ and assume that

$$
\beta=\omega^{\epsilon \cdot \omega+\beta_{1}}+\cdots+\omega^{\epsilon \cdot \omega+\beta_{r}}+\epsilon^{s} \cdot \beta_{r+1}+\cdots+\epsilon^{0} \cdot \beta_{r+1+s+1}
$$

where $\hat{\tau}>\beta_{1} \geq \ldots \geq \beta_{r}$ and $0<\beta_{r+1}$ and $\beta_{r+1}, \ldots, \beta_{r+1+s+1}<\epsilon$.

Then $\omega^{\beta_{1}}+\cdots+\omega^{\beta_{r}}<\omega^{\tau^{\prime}}$. By induction hypothesis there exists a quasiembedding $f: \omega^{\hat{\tau}} \rightarrow M(\tau)$. Let $f\left(\omega^{\beta_{1}}+\cdots+\omega^{\beta_{r}}\right)=\llbracket \delta_{1}, \ldots, \delta_{k} \rrbracket$.

In this situation we define

$$
e(\beta):=\llbracket \epsilon+\delta_{1}, \ldots, \epsilon+\delta_{k}, \beta_{r+1}, \beta_{r+1}+\beta_{r+2}, \ldots, \beta_{r+1}+\beta_{r+2}+\cdots+\beta_{n} \rrbracket .
$$

Then $e(\beta) \in M(\epsilon+\tau)$. Assume now that $e(\beta) \leq \diamond e(\gamma)$ where

$$
\beta=\omega^{\epsilon \cdot \omega+\beta_{1}}+\cdots+\omega^{\epsilon \cdot \omega+\beta_{r}}+\epsilon^{s} \cdot \beta_{r+1}+\cdots+\epsilon^{0} \cdot \beta_{r+1+s+1}
$$

and $\tau>\beta_{1} \geq \ldots \geq \beta_{r}$ and $0<\beta_{r+1}$ and $\beta_{r+1}, \ldots, \beta_{r+1+s+1}<\epsilon$ and

$$
\gamma=\omega^{\epsilon \cdot \omega+\gamma_{1}}+\cdots+\omega^{\epsilon \cdot \omega+\gamma_{t}}+\epsilon^{u} \cdot \gamma_{t+1}+\cdots+\epsilon^{0} \cdot \gamma_{t+1+u+1}
$$

where $\tau>\gamma_{1} \geq \ldots \geq \gamma_{t}$ and $0<\gamma_{t+1}, \ldots, \gamma_{t+1+u+1}<\epsilon$.

From $e(\beta) \leq e(\gamma)$ we obtain

$$
\llbracket \epsilon+\delta_{1}, \ldots, \epsilon+\delta_{k} \rrbracket \leq \llbracket \epsilon+\xi_{1}, \ldots, \epsilon+\xi_{l} \rrbracket \bmod M(\epsilon+\tau)
$$


hence $\llbracket \delta_{1}, \ldots, \delta_{k} \rrbracket \leq \llbracket \xi_{1}, \ldots, \xi_{l} \rrbracket \bmod M(\tau)$ thus

$$
f\left(\omega^{\beta_{1}}+\cdots+\omega^{\beta_{r}}\right) \leq f\left(\omega^{\gamma_{1}}+\cdots+\omega^{\gamma_{t}}\right) \bmod M(\tau)
$$

hence

$$
\omega^{\beta_{1}}+\cdots+\omega^{\beta_{r}} \leq \omega^{\gamma_{1}}+\cdots+\omega^{\gamma_{t}}
$$

and

$$
\omega^{\epsilon \cdot \omega+\beta_{1}}+\cdots+\omega^{\epsilon \cdot \omega+\beta_{r}} \leq \omega^{\epsilon \cdot \omega+\gamma_{1}}+\cdots+\omega^{\epsilon \cdot \omega+\gamma_{t}} .
$$

If strict inequality would hold then $\beta<\gamma$. So assume that

$$
\omega^{\epsilon \cdot \omega+\beta_{1}}+\cdots+\omega^{\epsilon \cdot \omega+\beta_{r}}=\omega^{\epsilon \cdot \omega+\gamma_{1}}+\cdots+\omega^{\epsilon \cdot \omega+\gamma_{t}} .
$$

Then

$\llbracket \beta_{r+1}, \beta_{r+1}+\beta_{r+2}, \ldots, \beta_{r+1}+\cdots+\beta_{r+1+s+1} \rrbracket \leq^{\diamond} \llbracket \gamma_{t+1}, \ldots, \gamma_{t+1}+\cdots+\gamma_{t+1+u+1} \rrbracket$.

Then necessarily $s \leq u$ since we need an injection for the embeddability. If $s<u$ then $\beta<\gamma$. So assume that $s=u$ and that $g$ is the injection witnessing $\llbracket \beta_{r+1}, \beta_{r+1}+\beta_{r+2}, \ldots, \beta_{r+1}+\cdots+\beta_{r+1+s+1} \rrbracket \leq^{\diamond} \llbracket \gamma_{t+1}, \ldots, \gamma_{t+1}+\cdots+\gamma_{t+1+u+1} \rrbracket$.

We claim that $\beta_{r+1} \leq \gamma_{t+1}$. Otherwise there would be an $m$ such that $\beta_{r+1}+$ $\cdots+\beta_{r+m} \leq \gamma_{t+1}$ under $g$. But then again $\beta_{r+1} \leq \gamma_{t+1}$. If now $\beta_{r+1}<\gamma_{t+1}$ then $\beta<\gamma$ and so assume that $\beta_{r+1}=\gamma_{t+1}$. Then the same $g$ also witnesses $\llbracket \beta_{r+1}+\beta_{r+2}, \ldots, \beta_{r+1}+\cdots+\beta_{r+1+s+1} \rrbracket \leq \llbracket \gamma_{t+1}+\gamma_{t+2}, \ldots, \gamma_{t+1}+\cdots+\gamma_{t+1+u+1} \rrbracket$.

Hence as before we may assume that

$$
\beta_{r+1}+\beta_{r+2}=\gamma_{t+1}+\gamma_{t+2}
$$

hence $\beta_{r+2}=\gamma_{t+2}$. This provides an easy inductive argument for proving $\beta \leq \gamma$.

As an application of our main theorem we obtain (following the proof of Corollary 4.2 in [1]) a refinement of a result of van den Dries and Ehrlich [5].

Corollary 1 Let $\Gamma$ be an ordered abelian group and $S \subset \Gamma^{\geq 0}$ well-ordered of order type $\alpha=o(S)$. Then the monoid generated by $S$ in $\Gamma$ is well-ordered of order type less than or equal to $\omega^{\hat{\alpha}}$.

Remarks: The calculation of $o(M(X))$ is not as simple as a corresponding calculation in [19]. There, to obtain a maximal linear extension, it was sufficient to extend $X$ to a (maximal) well-order and the induced multiset order was already maximal. In this paper the extension from $X$ to $o(X)$ leads only from an extension of the partial order $\leq^{\diamond}$ to another partial order $\preceq \diamond$ which still has to be linearized to produce the maximal linear extension. This phenomenon is familiar from Schmidt's calculation of maximal order types of several tree embeddability relations [15]. In her context it is also possible to construct (in a modular way) maximal linear extensions by first linearizing the sets of labels and then linearizing embeddability relations on trees with well-ordered sets of labels.

Acknowledgements: The author is grateful to Michael Rathjen and Antonio Montalban for inspiring discussions on the subject. The authors is grateful to the referees who provided helpful suggestions which led to an improved exposition. 


\section{References}

1. Aschenbrenner, M. and Pong, W.Y.: Orderings of monomial ideals. Fund. Math. 181, no. 1, 27-74 (2004)

2. Becker, T. and Weispfenning, V.: Gröbner bases. A computational approach to commutative algebra. In cooperation with Heinz Kredel. Graduate Texts in Mathematics, 141. Springer-Verlag, New York (1993)

3. Buchholz, W., Cichon, E.A. and Weiermann, A.: A uniform approach to fundamental sequences and hierarchies. Math. Logic Quart. 40, no. 2, 273-286 (1994)

4. De Jongh, D.H.J. and R. Parikh, R.: Well-partial orderings and hierarchies. Nederl. Akad. Wetensch. Proc. Ser. A 80=Indag. Math. 39, no. 3, 195-207 (1977)

5. Van den Dries, L. and Ehrlich, P.: Fields of surreal numbers an exponentiation. Fund. Math. 168, 2, (2001), 173-188 and Erratum to [5] in Fund. Math. 167, 3, 295-297 (2001)

6. N. Dershowitz N. and Jouannaud, J.P.: Rewrite systems. In: Handbook of Theoretical Computer Science, Part B, Elsevier, 243-320 (1990)

7. Ehrenfeucht, A.: Polynomial functions with exponentiation are well-ordered. Algebra Universalis 3, 261-262 (1973)

8. Feferman, S.: Systems of predicative analysis. II. Representations of ordinals. J. Symbolic Logic 33, 193-220 (1968)

9. Gallier, J.H.: What's so special about Kruskal's theorem and the ordinal $\Gamma_{0}$ ? A survey of some results in proof theory. Annals of Pure and Applied Logic 53 199-260 (1991)

10. Girard, J.Y.: $\Pi_{2}^{1}$-logic. I. Dilators. Ann. Math. Logic 21 (1981), no. 2-3, 75-219 (1982)

11. Hasegawa, R.: Two applications of analytic functors. Theories of types and proofs (Tokyo, 1997). Theoret. Comput. Sci. 272, no. 1-2, 113-175 (2002)

12. Montalbán, A.: Computable linearizations of well-partial-orderings. Order 24, no. 1, 39-48 (2007)

13. Rathjen, M. and Weiermann, A.: Proof-theoretic investigations on Kruskal's theorem. Annals of Pure and Applied Logic 60, 49-88 (1993)

14. Pohlers, W.: Proof Theory. The first step into Impredicativity. Springer, Berlin (2009)

15. Schmidt, D.: Well-Partial Orderings and Their Maximal Order Types. Habilitationsschrift, Heidelberg (1979)

16. Schütte, K. and Simpson, S.G.: Ein in der reinen Zahlentheorie unbeweisbarer Satz über endliche Folgen von natürlichen Zahlen. Archiv für mathematische Logik und Grundlagenforschung 25, 75-89 (1985)

17. Simpson, S.G.: Nonprovability of certain combinatorial properties of finite trees. In: Harvey Friedman's research on the foundations of mathematics, 87-117, Stud. Logic Found. Math., 117, North-Holland, Amsterdam (1985)

18. Urquhart, A.: The complexity of decision procedures in relevance logic. II. J. Symbolic Logic 64 no. 4, 1774-1802 (1999)

19. Weiermann, A.: Proving termination for term rewriting systems. Proceedings of CSL 91. LNCS 626 419-428 (1992)

20. Weiermann, A.: An order-theoretic characterization of the Schütte-Veblenhierarchy. Math. Logic Quart. 39 no. 3, 367-383 (1993)

21. Weiermann, A.: Well partial orderings and their strengths measured in maximal order types. Summary of a talk given at the conference on computability, reverse mathematics and combinatorics in Banff 2008 